\title{
Dual redshift on Planck-scale-curved momentum spaces
}

\author{
Giovanni AMELINO-CAMELIA, ${ }^{1,2}$ Leonardo BARCAROLI, ${ }^{1,2}$ Giulia GUBITOSI, ${ }^{1,2}$ and Niccoló LORET ${ }^{1,2}$ \\ ${ }^{1}$ Dipartimento di Fisica, Università di Roma "La Sapienza", P.le A. Moro 2, 00185 Roma, Italy \\ ${ }^{2}$ INFN, Sez. Roma1, P.le A. Moro 2, 00185 Roma, Italy
}

Several approaches to the investigation of the quantum-gravity problem have provided "theoretical evidence" of a role for the Planck scale in characterizing the geometry of momentum space. One of the main obstructions for a full exploitation of this scenario is the understanding of the role of the Planck-scale-curved geometry of momentum space in the correlations between emission and detection times, the "travel times" for a particle to go from a given emitter to a given detector. These travel times appear to receive Planck-scale corrections for which no standard interpretation is applicable, and the associated implications for spacetime locality gave rise to the notion of "relative locality" which is still in the early stages of investigation. We here show that these Planck-scale corrections to travel times can be described as "dual redshift" (or "lateshift"): they are manifestations of momentum-space curvature of the same type already known for ordinary redshift produced by spacetime curvature. In turn we can identify the novel notion of "relative momentum-space locality" as a known but under-appreciated feature associated to ordinary redshift produced by spacetime curvature, and this can be described in complete analogy with the relative spacetime locality that became of interest in the recent quantum-gravity literature. We also briefly comment on how these findings may be relevant for an approach to the quantum-gravity problem proposed by Max Born in 1938 and centered on Born duality. 


\section{INTRODUCTION}

Over the last decade several independent arguments pointed more or less explicitly toward a role for the Planck scale in characterizing a non-trivial geometry of momentum space (see, e.g., Refs. [1 8]). Remarkably Max Born had already argued in 1938 [9], inspired by Born duality, that curvature of momentum space might be a needed step toward quantum gravity, but for several decades this had met little interest (see, however, Ref. 10]). Attention to this scenario was imposed by some of the most modern formalisms in use for the study of the quantum-gravity problem. For example, in the study of noncommutative spacetimes, particularly when considering models with "Lie-algebra spacetime noncommutativity", $\left[x_{\mu}, x_{\nu}\right]=i \zeta_{\mu \nu}^{\sigma} x_{\sigma}$, the momentum space on which spacetime coordinates generate translations is evidently curved (see, e.g., Ref [11]). And also in the study of the Loop Quantum Gravity approach 12 one can adopt a perspective involving momentum-space curvature (see, e.g., Ref [13]).

In light of these results, it could be important for quantum-gravity research to identify the main observable consequences of momentum-space curvature. And indeed there has been a strong effort recently concerning what appears to be the best candidate manifestation of Planck-scale momentum-space curvature: these are studies [11, 14 19] of the correlations between emission and detection times, the "travel times" between a given emitter and a given detector. It is found that the Planckscale curvature of momentum space introduces corrections to the travel times, opening also an opportunity for experimental tests [11, 14 18. But the conceptual status of these Planck-scale corrections to travel times remains only poorly understood, and in particular it is emerging that a crucial role should be played by a correspondingly weaker notion of spacetime locality, the "relative locality" of Refs. [7, 8, 20, 24] whose understanding is just in the early stages of development.

We here show that these gray areas of our understanding of corrections to travel times due to Planck-scale curvature of momentum space and the associated relativity of spacetime locality can be clarified by fully embracing the spirit of Born's 1938 proposal. These aspects of the implications of curvature of momentum space must admit a description which is just dual to known properties of theories with spacetime curvature. And indeed we find that the travel-time features noticed in studies with momentum-space curvature admit description as "dual redshift", which we shall here also label as "lateshift": they are manifestations of momentum-space curvature of the same type already known for ordinary redshift produced by spacetime curvature.
In order to keep our presentation clear and compact we focus on the case of only $1+1$ spacetime dimensions $(2 D)$, investigating the duality between 2D de Sitter spacetime (dS spacetime) and 2D de Sitter momentum space (dS momentum space).

We derive very explicitly a description of the corrections to travel times due to the Planck-scale curvature of momentum space given in terms of "dual redshift". And also for relative spacetime locality produced by momentum-space curvature we find that it is dual to a known but under-appreciated feature associated to ordinary redshift produced by spacetime curvature, which we here label "relative momentum-space locality". The presence of relative spacetime locality for theories with curved momentum space has been occasionally perceived with an aura of mystique and/or suspicion (see, e.g., Ref. 25, 26]), but our analysis clarifies that relative locality is a simple and intelligible consequence of cases in which the observers cannot or anyway do not adopt spacetime coordinates that are conjugate to the generators of spacetime translations ${ }^{1}$. And in fact we can point the attention of our readers to a well-known dual feature, which we feel deserves to be labeled as "relative momentum-space locality", which is present in the classic analyses of de Sitter spacetime and reflects indeed the fact that the curvature of de Sitter spacetime can encourage the adoption of coordinates on momentum space that are not conjugate to spacetime coordinates.

\section{FROM REDSHIFT TO LATESHIFT}

We start by essentially summarizing our key results for lateshift, sketching out the duality that emerges from our analysis between the lateshift produced by momentumspace curvature and ordinary redshift produced by spacetime curvature. Later sections will provide further details. We illustrate the duality by using comoving coordinates on the 2D-dS-spacetime side and for the $2 \mathrm{D}-\mathrm{dS}$ momentum space we use coordinates dual to those ("comoving on momentum space"). The duality is centered on exchanging the expansion rate $H$ of $\mathrm{dS}$ spacetime for the inverse of the Planck scale, here denoted by $\ell$, which plays indeed the formal role of expansion rate on the dS momentum-space side of the duality.

\footnotetext{
1 We here focus on spacetime translations, but the careful reader will appreciate that the argument generalizes to any case in which the coordinates have nontrivial properties under the transformations of interest. For example, the analysis in Ref. 20] considers a curved momentum space and a pair of distantly boosted observers (connected by a boost in combination with a translation), and there the relative locality arises because the spacetime coordinates do not have simple properties under the action of compositions of boosts and translations.
} 


\section{A. Redshift}

For dS 1 + 1-dimensional spacetime the metric takes the form $(\mu, \nu=0,1)$

$$
d s^{2}=\left(d x^{0}\right)^{2}-e^{2 H x^{0}}\left(d x^{1}\right)^{2}
$$

while energy $p_{0}$ and spatial momentum $p_{1}$ are conjugate to the spacetime coordinates:

$$
\left\{x^{0}, x^{1}\right\}=0, \quad\left\{p_{0}, p_{1}\right\}=0, \quad\left\{p_{\mu}, x^{\nu}\right\}=\delta_{\mu}^{\nu} .
$$

Spatial momentum is a conserved charge, for which we shall use equivalently the notation $p_{1}$ and $\Pi_{1}$. Energy is not conserved, because of spacetime expansion. Time translations are deformed by spacetime expansion, and the associated charge $\Pi_{0}$ has the properties

$\left\{\Pi_{0}, \Pi_{1}\right\}=H \Pi_{1}, \quad\left\{\Pi_{0}, x^{1}\right\}=-H x^{1}, \quad\left\{\Pi_{0}, x^{0}\right\}=1$.

It is useful to notice that $\Pi_{0}=p_{0}-H x^{1} p_{1}$. And it shall be relevant for rendering more vivid our duality to observe that in dS spacetime (with comoving coordinates) the worldlines of massless particles crossing the origin of the observer take the form

$$
x^{1}=\frac{1-e^{-H x^{0}}}{H}
$$

For a particle on such a worldline one has that energy and momentum are related through the particle's time coordinate:

$$
p_{1}=-e^{H x^{0}} p_{0}
$$

The conceptual content of redshift in dS spacetime is particularly intuitive when comparing results for energy measurements by two observers, say Alice and Bob, whose origins are connected by a worldline of type (4). Indeed redshift is an effect such that a blue particle emitted at some source reaches a distant telescope as a red particle. For easier comparison with the results we shall later derive for a curved momentum space, we prefer to characterize quantitatively the redshift effect due to spacetime curvature by comparing its effects on two different particles emitted with the same energy ("both blue") and from the same source but at different times. We therefore consider two particles emitted with the same energy by emitter Alice (the two worldlines both cross Alice's spatial origin) and derive in Sec. IV the difference in energy of detection of these two particles at some distant detector Bob (Bob is such that the two particles both cross Bob's spatial origin). We find the following result:

$$
\left\{\begin{array}{l}
\tilde{p}_{0}^{@ A}=p_{0}^{@ A}, \quad \tilde{x}_{@ A}^{0} \neq x_{@ A}^{0} \\
\tilde{p}_{0}^{@ B}=e^{-H\left[\tilde{x}_{@ B}^{0}-x_{@ B}^{0}\right]} p_{0}^{@ B}
\end{array}\right.
$$

where $p_{0}^{@ B}$ and $\tilde{p}_{0}^{@ B}$ (respectively $x_{@ B}^{0}$ and $\tilde{x}_{@ B}^{0}$ ) are the energies (respectively the times) of detection at Bob, indeed for two particles emitted at Alice with the same energy $\left(\tilde{p}_{0}^{@ A}=p_{0}^{@ A}\right)$ but at different times $\left(\tilde{x}_{@ A}^{0} \neq x_{@ A}^{0}\right)$.

\section{B. Lateshift}

In establishing the duality with the results in the previous subsection we of course describe the metric of $(1+1$ dimensional) dS momentum space as follows:

$$
d k^{2}=\left(d p_{0}\right)^{2}-e^{2 \ell p_{0}}\left(d p_{1}\right)^{2}
$$

And we introduce spacetime coordinates as conjugate to the momenta:

$$
\left\{x^{1}, x^{0}\right\}=0, \quad\left\{p_{1}, p_{0}\right\}=0, \quad\left\{x^{\mu}, p_{\nu}\right\}=\delta_{\nu}^{\mu},
$$

We shall keep the analogy as close as possible by also introducing (in analogy with $\Pi_{0}, \Pi_{1}$ of the previous subsection) some "relative-locality coordinates" $\chi^{0}, \chi^{1}$ with $\chi^{1} \equiv x^{1}$ and $\chi^{0} \equiv x^{0}-\ell x^{1} p_{1}$, so that

$$
\left\{\chi^{0}, \chi^{1}\right\}=\ell \chi^{1}, \quad\left\{\chi^{0}, p_{1}\right\}=-\ell p_{1}, \quad\left\{\chi^{0}, p_{0}\right\}=1 .
$$

$\chi^{0}$ and $\chi^{1}$ generate the translational symmetries of the dS momentum space, but (again in analogy with the dS spacetime case) $\chi^{0}$ does not generate pure $p_{0}$ shifts.

We shall show that the on-shell condition for massless particles on the dS momentum-space takes the form

$$
p_{1}=\frac{1-e^{-\ell p_{0}}}{\ell},
$$

which is interestingly dual to the Eq. (4) for the worldlines of massless particles in dS spacetime, while the dSmomentum-space picture of worldlines of massless particles is given by

$$
x^{1}=-e^{\ell p_{0}} x^{0}
$$

which is interestingly dual to the Eq. (5) playing the role of on-shell relation on the dS-spacetime side.

These Eqs. (7), (8), (9), (10), (11) are exactly dual to the Eqs.(1), (2), (3), (4), (5) valid on the dS spacetime. This will prove sufficient for our purposes even though there is an element of our analysis that is not properly dual: properties of spacetime translations are responsible for both redshift and lateshift. An even more precise duality would be found if one studied the implications of momentum-space curvature for momentum-space translations, but those are of limited interest in physics. The duality between redshift and lateshift is nonetheless strong enough to allow us to derive in Sec V a result, which we propose as main characterization of lateshift, which is indeed dual to the characterization of redshift we gave in Eq. (6). This is found by considering again an emitter Alice and a detector Bob, and takes the shape of the relationship

$$
\left\{\begin{array}{l}
\tilde{p}_{0}^{@ A} \neq p_{0}^{@ A}, \tilde{x}_{@ A}^{0}=x_{@ A}^{0} \\
\tilde{x}_{@ B}^{0}-\tilde{x}_{@ A}^{0}=e^{-\ell\left[\tilde{p}_{0}^{@ B}-p_{0}^{@ B}\right]}\left(x_{@ B}^{0}-x_{@ A}^{0}\right),
\end{array}\right.
$$

where, consistently with the duality we are exposing, for this result 12 we consider two particles emitted at the 
same time at Alice $\left(\tilde{x}_{@ A}^{0}=x_{@ A}^{0}\right)$ with different energies $\left(\tilde{p}_{0}^{@ A} \neq p_{0}^{@ A}\right)$.

This result 12 evidently characterizes lateshift as the source of the peculiarities for the correlations between emission times and detection times previously found in the curved-momentum-space literature: Eq. (12) confirms that in presence of momentum-space curvature two massless particles emitted simultaneously at Alice with different energies $\left(\tilde{p}_{0}^{@ A} \neq p_{0}^{\circledR A}\right)$ reach a distant detector Bob at different times $\left(\tilde{x}_{@ B}^{0} \neq x_{@ B}^{0}\right)$, indeed governed by (12).

We stress again that the relativistic duality between the two cases is exact: formulas (1), (2), (3), (4), (5) familiar for dS spacetime get mapped into the exactly dual formulas $(7),(8),(9),(10),(11)$ for the novel case of dS momentum space. But the questions we typically ask experimentally to these exactly dual pictures are not exactly dual to each other: in both cases one is interested in spacetime translations, since in both cases one is primarily considering situations with spatially distant emitter and detector. The relativistic duality we are exposing is however so strong that it still affects very significantly the final results (6) and 12 .

It is also useful to observe that the duality we are analyzing becomes trivial when no curvature is present: relativistic theories of Minkowski spacetime and relativistic theories of Minkowski momentum space coincide (our duality turns into a self-duality when curvature is absent). In the Minkowski case massless particles with any difference in energy $\tilde{p}_{0}^{@ A}-p_{0}^{@ A}$ emitted with any emissiontime difference $x_{@ A}^{0}-\tilde{x}_{@ A}^{0}$ at emitter Alice are then detected at some distant detector Bob (at rest with respect to the emitter) with same difference of detection times $x_{@ B}^{0}-\tilde{x}_{@ B}^{0}=x_{@ A}^{0}-\tilde{x}_{@ A}^{0}$ and the same difference of energies $\tilde{p}_{0}^{\mathrm{Q} B}-p_{0}^{\mathrm{Q} B}=\tilde{p}_{0}^{\mathrm{Q} A}-p_{0}^{@ A}$.

In Figs 1 and 2 we visualize an aspect of the duality here exposed for the case when curvature is present, also in reference to the self-duality present when there is no curvature. For these visualization purposes we find useful to rely on the correlations that the analysis we present in later sections finds between time of detection at Bob and energy of detection at Bob of a massless particle. The presence of a horizontal dotted line in both Fig 1 and Fig 2 reflects the fact that in absence of curvature the energy of detection is independent of the time of detection (in absence of curvature there is no redshift, so the energy of detection is automatically the same as the energy of emission of the particle) and the time of detection is independent of the energy of detection (in absence of curvature there is no lateshift, so the time of detection is given, for massless particles of any energy, in terms of the distance between emitter and detector).

Fig.1 also shows (solid line) the quantitative behaviour of redshift produced by spacetime curvature: for fixed time and energy of emission at Alice there is a correlation (governed by the distance between Alice and Bob, left implicit in figure) between the time of detection at Bob and the energy of detection at Bob. This correlation is of course governed by the distance between Alice and Bob, left implicit in figure (but notice that the graph does indicate the value of energy for $x_{@ B}^{0}=0$ which is the case with Alice as both the emitter and the detector, i.e. no distance between emitter and detector). For the case of de Sitter expansion this gives indeed lower values of detection energy at higher values of detection time.

And Fig 2 also shows (solid line) the quantitative behaviour of lateshift produced by momentum-space curvature. Here too lower values of detection energy are found at higher values of detection time, but, as here shown in Sec $\mathrm{V}$ the exponential law governing these correlations takes form dual to the corresponding exponential law found for the spacetime-curvature case.

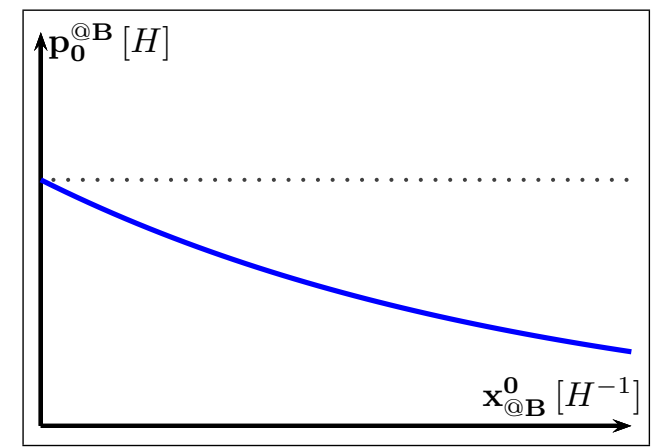

Figure 1. We here show how, for fixed time and energy of emission at Alice, there is a correlation between the time of detection at Bob and the energy of detection at Bob, for the case of Minkowskian spacetime (dotted line) and the case of dS spacetime (solid line). The behaviour here shown for the dS-spacetime case, which is a characteristic manifestation of redshift, is governed by Eq. 52 here derived in the later SecIV

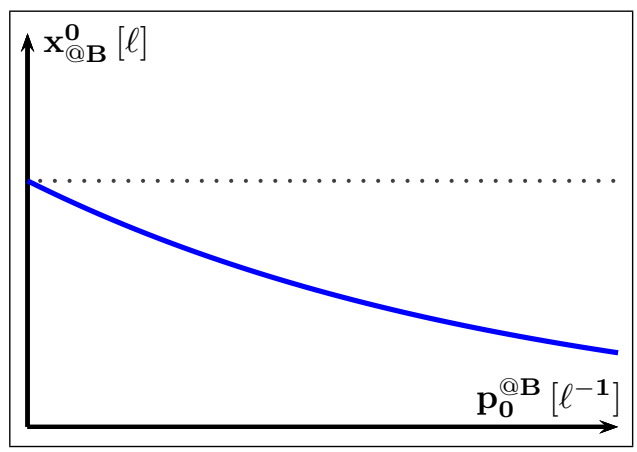

Figure 2. We here show how, for fixed time and energy of emission at Alice, there is a correlation between the time of detection at Bob and the energy of detection at Bob, for the case of Minkowskian momentum space (dotted line) and the case of dS momentum space (solid line). The behaviour here shown for the dS-momentum-space case, which is a characteristic manifestation of lateshift, is governed by Eq. 91 here derived in the later Sec V

\section{TWO TYPES OF RELATIVE LOCALITY}

A striking aspect of some of the studies triggered by the recent interest in Planck-scale curved momentum space is 
the one concerning relative spacetime locality. It is found that in a variety of contexts the requirement of locality of physical theories must be weakened in presence of momentum-space curvature. One still insists that events should be local, but allows for the possibility that the locality be manifest only in the coordinatizations of the event given by nearby observers. The inferences about the event made by distant observers (according to the coordinatizations they adopt) may not manifest the locality of the event witnessed by nearby observers. So this weaker principle of locality allows for a relative notion of spacetime locality to replace the ordinary absolute (observer-indepedent) notion of locality.

In this section we shall summarize our results (later described in greater detail) showing that even just for free particles on a de Sitter momentum space observers could naturally adopt coordinates such that relative spacetime locality is present. This will establish a connection between the sort of framework we are here considering and the presence of relative spacetime locality found in previous studies based on various aspects of curvature of momentum space, such as [16, 20, 23, 24, 27.

And still in this section we shall also revisit briefly classic results on the implications of spacetime curvature which (when analyzed on the background of the recent interest in relative spacetime locality) deserve to be labelled as effects of "relative momentum-space locality": once again there is a duality between the relativity of spacetime locality produced by a de Sitter momentum space and the relative momentum-space locality produced by a de Sitter spacetime.

\section{A. Relative spacetime locality from momentum-space curvature}

In our simple framework of free particles on a de Sitter momentum space the opportunity for discussing relative spacetime locality comes from the option of choosing between the coordinates $x^{0}, x^{1}$ and the coordinates $\chi^{0}, \chi^{1}$. For the discussion of lateshift given in the previous section we relied on the coordinates $x^{0}, x^{1}$ which are free from relative-locality features, but it should be noticed that those coordinates do not generate translations on momentum space (so they miss one of the defining properties that spacetime coordinates enjoy when momentum space has no curvature). The coordinates $\chi^{0}, \chi^{1}$ do generate translations on our curved momentum space but are affected, as we shall now see, by relative-locality features.

It is useful to note down again the relationship between $x^{0}, x^{1}$ and $\chi^{0}, \chi^{1}$ coordinates,

$$
\begin{gathered}
\chi^{1}=x^{1} \\
\chi^{0}=x^{0}-\ell x^{1} p_{1}
\end{gathered}
$$

and to also note here some of the results derived in later sections when working with the $x^{0}, x^{1}$ coordinates:
- with $x^{0}, x^{1}$ coordinates the worldlines of massless particles of energy-momentum $p^{0}, p^{1}$ have the form

$$
x^{1}-\bar{x}^{1}=\frac{p^{1}}{\left|p^{1}\right|} e^{\ell p^{0}}\left(x^{0}-\bar{x}^{0}\right),
$$

- the coordinate transformations between observers Alice and Bob connected by a pure translation of parameters $a^{0}, a^{1}$ are

$$
\begin{aligned}
& x_{B}^{0}=x_{A}^{0}-a^{0} \\
& x_{B}^{1}=x_{A}^{1}-a^{1} .
\end{aligned}
$$

- with $\chi^{0}, \chi^{1}$ coordinates the worldlines of massless particles of energy-momentum $p^{0}, p^{1}$ have the form

$$
\chi^{1}-\bar{\chi}^{1}=\frac{p^{1}}{\left|p^{1}\right|}\left(\chi^{0}-\bar{\chi}^{0}\right),
$$

- and the coordinate transformations between observers Alice and Bob connected by a pure translation of parameters $a^{0}, a^{1}$ are

$$
\begin{aligned}
& \chi_{B}^{0}=\chi_{A}^{0}-a^{0}+a^{1} \ell p_{1} \\
& \chi_{B}^{1}=\chi_{A}^{1}-a^{1} .
\end{aligned}
$$

On the basis of these observations one already gets a rather clear picture of the situation: on one side, with $x^{0}, x^{1}$ coordinates, the curvature of momentum space affects the form of the worldline (producing an energydependent velocity) but leaves the translation transformations unaffected, while on the other side, with $\chi^{0}, \chi^{1}$ coordinates, one has the opposite situation of worldlines unaffected by the curvature of momentum space but with translation transformations that reflect momentum space curvature.

As already stressed in previous studies of relative spacetime locality these apparently alternative pictures of the same physical system are ultimately found to agree on the "true observables" of such theories which are times of emission or detection "at observers" (in the spatial origin of the observer). But for this consistency for observables obtained within the two alternative coordinatizations an important role is played by relative spacetime locality.

To see this it is convenient to contemplate the case of two particles of different energy emitted simultaneously at Alice toward Bob. Adopting the $x^{0}, x^{1}$ coordinates, with a momentum dependent coordinate velocity, one evidently then finds that the times of arrival at Bob of the two particles are different. This of course is nothing else but the lateshift we already described above. When using the $\chi^{0}, \chi^{1}$ coordinates to describe the same situation one finds that according to Alice the times of arrival at Bob are identical, since the coordinate velocity in the coordinatization $\chi^{0}, \chi^{1}$ is momentum independent. But with $\chi^{0}, \chi^{1}$ coordinates one must take into account relative locality, i.e. the fact that translation transformations are affected by momentum space curvature. And the result 
is that while according to Alice the particles reach Bob simultaneously, actually according to Bob (an observer near the detections) the detections are not simultaneous: what Bob finds using $\chi^{0}, \chi^{1}$ coordinates is a difference of detection times that reproduces exactly the difference of detection times at Bob obtained with $x^{0}, x^{1}$ coordinates. So one has the same final result for the lateshift effect using both types of coordinates, even though some aspects of the analysis do change. The point is that the two choices of coordinatization always agree on which were the times of emission at Alice according to Alice and which were the detection times at Bob according to Bob. All this is summarized in Fig. 3.

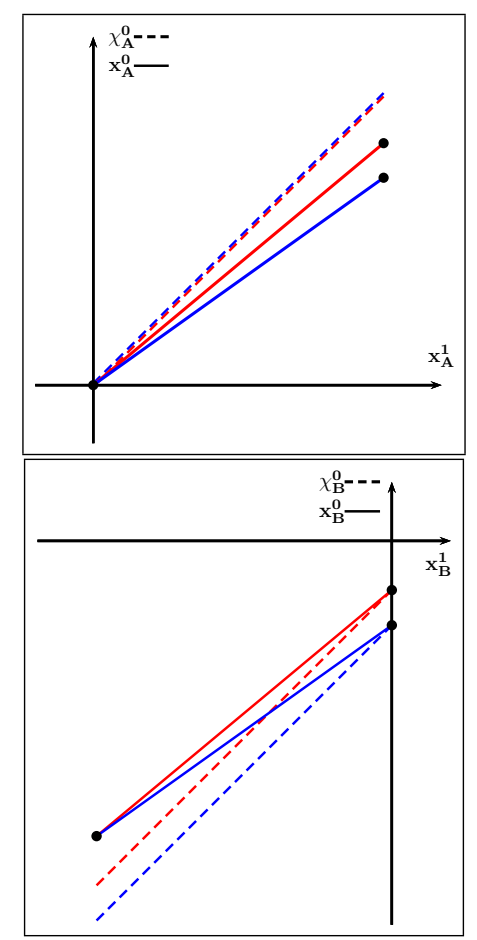

Figure 3. We here visualize our preferred illustrative example of a relative-spacetime-locality analysis. Alice's descriptions of massless-particle worldlines are in the top panel, while Bob's descriptions of the same worldlines are in the bottom panel. Both with $x^{0}, x^{1}$ coordinates (solid lines) and with $\chi^{0}, \chi^{1}$ coordinates (dashed lines) Alice has that the emission is simultaneous and Bob has that the detections occur at different times (same time difference within both coordinatizations). The points marked on the solid lines identify the values of $\left\{p_{0}, x^{0}\right\}$ measured at Alice and at Bob. The peculiarities introduced by the coordinatization $\chi^{0}, \chi^{1}$, affected by relative spacetime locality, only play a role in the inferences the observers make about distant events: adopting $\chi^{0}, \chi^{1}$ coordinates Alice's would describe the distant detections at Bob as simultaneous and Bob would describe the distant emissions at Alice as not simultaneous. Equations in support of these figures will be derived in Sec $\nabla$

\section{B. Momentum-space relative locality from spacetime curvature}

Our next task is to expose the fact that relative locality is also produced by spacetime curvature: we find that momentum-space locality is relative when adopting one of the natural choices of momentum-space coordinates for the dS-spacetime case. The choice of coordinatization of momentum space where the curvature of dS spacetime produces relative-momentum-space-locality effects was here already introduced earlier: it is the one with coordinatization of momentum space given by $\Pi_{0}, \Pi_{1}$, the conserved charges generating translations of de Sitter spacetime. We note down again here their relationship to the coordinatization given in terms of energy $p_{0}$ and spatial momentum $p_{1}$, canonically conjugate to the spacetime coordinates:

$$
\begin{aligned}
& \Pi_{0}=p_{0}-H x^{1} p_{1} \\
& \Pi_{1}=p_{1}
\end{aligned}
$$

Again it is useful to characterize the relative-locality effects by considering two observers, Alice and Bob, on the worldline of a massless particle (the particle crosses the spacetime origins of both Alice and Bob). In the dS spacetime two such observers are connected by a translation with translations parameters linked simply by

$$
a^{0}=a^{1}
$$

For such observers the charges $\Pi_{0}, \Pi_{1}$, conserved along the worldline of the massless particle, must satisfy ${ }^{2}$

$$
\Pi_{0}=-\Pi_{1}
$$

But while both Alice and Bob agree on this relation, they attribute different values to these charges: the translation transformations of $\mathrm{dS}$ spacetime act non trivially on the charges $\Pi_{0}$ and $\Pi_{1}$,

$$
\left\{\Pi_{0}, \Pi_{1}\right\}=H \Pi_{1}
$$

and the net result is the following relationship between the values attributed to the charges by Alice and by Bob:

$$
\begin{aligned}
& \Pi_{0}^{B}=\Pi_{0}^{A}+\Pi_{1}^{A}\left(1-e^{-H a^{0}}\right) \\
& \Pi_{1}^{B}=e^{-H a^{0}} \Pi_{1}^{A}
\end{aligned}
$$

These relationships are the essence of the relative momentum-space locality caused by spacetime curvature which we here want to highlight. It is useful to the understanding of this feature to see how the key aspects of

\footnotetext{
${ }^{2}$ We are specifying our analysis to the case of negative $\Pi_{1}$ which gives the case of particle emitted at Alice and propagating toward Bob along the positive $x$-direction. (The case of positive $\Pi_{1}$, i.e. $\Pi_{1}=\Pi_{0}$ is equally interesting, but of course gives rise to exactly the same qualitative picture.)
} 
the $\Pi_{0}, \Pi_{1}$ coordinatization get described if instead one uses the more customary $p_{0}, p_{1}$ coordinatization. A key point in this respect is that the result $\Pi_{0}=-\Pi_{1}$ gets converted into

$$
p_{0}=-p_{1} e^{-H x^{0}},
$$

and the transformation laws $(19)$ and $(20)$ get converted into

$$
\begin{aligned}
& p_{0}^{B}=p_{0}^{A} \\
& p_{1}^{B}=p_{1}^{A} e^{-H a^{0}} .
\end{aligned}
$$

Evidently the key aspect for our analysis of relative momentum-space locality is the comparison of $(19)$ and (22) and how those differences exactly compensate the differences between (18) and (21). To see this it is convenient to contemplate the case of two particles which according to Alice have the same energy but are emitted at different times toward Bob. Adopting the $p^{0}, p^{1}$ coordinates, with trivial transformation (22) of $p^{0}$, Alice sees redshift as an effect encoded fully in (21), which in particular is such that the energies at Bob are different (because of the different emission times) even though the energies at Alice are the same. If Bob also adopts the $p^{0}, p^{1}$ coordinates he gets a picture completely consistent with Alice's, since with the $p^{0}, p^{1}$ coordinates there is no relative momentum-space locality.

If instead Alice and Bob adopt the $\Pi_{0}, \Pi_{1}$ coordinatization, affected by relative locality, the same physical picture is described in a different way. Both Alice and Bob have $\Pi_{0}$ as a conserved charge, but the value Alice gives to $\Pi_{0}$ is different from the value Bob gives to $\Pi_{0}$, as specified by $(19)$. The mismatch between $\Pi_{0}$ according to Alice and $\Pi_{0}$ according to Bob depends on the timetranslation parameter that connects Alice to Bob, so the fact that the particles are emitted at different times at Alice renders them differently subject to redshift.

The net result is that the pictures given by the $p^{0}, p^{1}$ coordinates and by $\Pi_{0}, \Pi_{1}$ coordinates are intuitively consistent with each other for what concerns the values of energy measured (and the times of those measurements) at both Alice and Bob. The only mismatches between the pictures with $p^{0}, p^{1}$ coordinates and the picture with $\Pi_{0}, \Pi_{1}$ coordinates concerns inferences about energies at distant emission/detection events: those inferences are misleading when adopting the $\Pi_{0}, \Pi_{1}$ coordinates. Alice witnesses emissions of particles with the same $\Pi_{0}$, but Bob infers that those distant emissions were with different $\Pi_{0}$. And similarly Bob witnesses detections of particles with different $\Pi_{0}$, but Alice infers that those distant detections are at the same $\Pi_{0}$.

All this is summarized in Fig. 4.

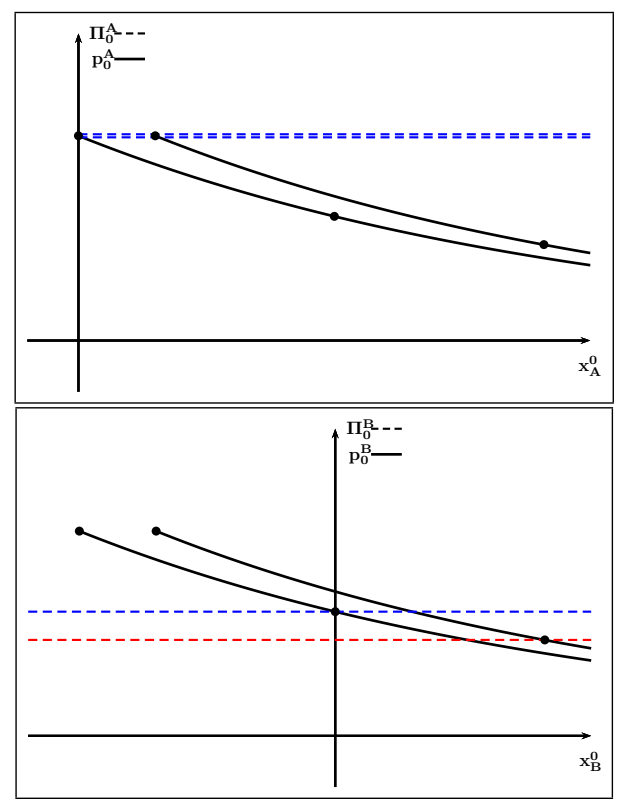

Figure 4. We here visualize our preferred illustrative example of a relative-momentum-space-locality analysis, which concerns the evolution in time of $\Pi_{0}$ and $p_{0}$ on the worldlines of massless particles. Alice's descriptions of some worldlines are in the top panel, while Bob's description of the same worldlines are in the bottom panel. Both with $p_{0}, p_{1}$ coordinates (solid lines) and with $\Pi_{0}, \Pi_{1}$ coordinates (dashed lines) Alice has that the emitted particles have the same energy and Bob has that the energies at detection are different (same energy difference within both coordinatizations). The peculiarities introduced by the coordinatization $\Pi_{0}, \Pi_{1}$, affected by relative momentum-space locality, only play a role in the inferences the observers make about distant events: adopting $\Pi_{0}, \Pi_{1}$ coordinates Alice would describe the distant detections at Bob as having the same energy and Bob would describe the distant emissions at Alice as having different energy. Equations in support of these figures will be derived in Sec IV

\section{DE SITTER SPACE-TIME}

In the previous sections we summarized the key points of our thesis, establishing a duality between redshift in dS spacetime and lateshift in dS momentum space, and establishing how this duality also affects the associated relative-locality effects. As announced we shall now provide more detailed derivations of those key points of our thesis. We start in this section with the dS-spacetime case.

We start by noticing that in comoving coordinates the dS-spacetime metric, from which we obtain the spacetime interval (1), and its inverse can be represented by the matrices

$$
g_{\mu \nu}=\left(\begin{array}{cc}
1 & 0 \\
0 & -e^{2 H x^{0}}
\end{array}\right), \quad g^{\mu \nu}=\left(\begin{array}{cc}
1 & 0 \\
0 & -e^{-2 H x^{0}}
\end{array}\right)
$$

The physical momentum $p^{\mu}$ of a particle of mass $m$, measured by a free-falling observer, is

$$
p^{\mu}=m \dot{x}^{\mu}
$$

where (as we shall do consistently) the dotted coordi- 
nate $\dot{x}^{\mu}$ is differentiated with respect a worldline affine parameter $\tau$, so that $\dot{x}^{\mu} \equiv d x^{\mu} / d \tau$. In the following we will mainly work with their lowered-index version,

$$
p_{0}=p^{0}, \quad p_{j}=-\delta_{i}^{j} e^{2 H x^{0}} p^{i},
$$

which satisfy ordinary Poisson algebra with coordinates:

$$
\begin{aligned}
& \left\{p_{0}, x^{0}\right\}=1, \quad\left\{p_{0}, x^{1}\right\}=0, \\
& \left\{p_{1}, x^{0}\right\}=0, \quad\left\{p_{1}, x^{1}\right\}=1,
\end{aligned}
$$

We use standard notation for Poisson brackets

$$
\{A, B\}=\omega_{a b} \frac{\partial A}{\partial \xi^{a}} \frac{\partial B}{\partial \xi^{b}}
$$

where $\xi^{a}$ are the phase space coordinates, and $\omega_{a b}$ identifies the phase space symplectic structure.

The conserved charges associated with the translation and boost transformations we used in section III B have the following representation on coordinates and momenta:

$$
\begin{gathered}
\Pi_{0}=p_{0}-H x^{1} p_{1}, \quad \Pi_{1}=p_{1}, \\
N=x^{1} p_{0}+\left(\frac{1-e^{-2 H x^{0}}}{2 H}-\frac{H}{2}\left(x^{1}\right)^{2}\right) p_{1} .
\end{gathered}
$$

We can also re-express the boost charge in terms of translation-transformation charges $\Pi_{\alpha}$ :

$$
N=x^{1} \Pi_{0}+\left(\frac{1-e^{-2 H x^{0}}}{2 H}+\frac{H}{2}\left(x^{1}\right)^{2}\right) \Pi_{1} .
$$

These charges satisfy the algebra

$$
\begin{gathered}
\left\{\Pi_{0}, \Pi_{1}\right\}=H \Pi_{1}, \quad\left\{N, \Pi_{1}\right\}=-\Pi_{0} \\
\left\{N, \Pi_{0}\right\}=-\Pi_{1}+H N,
\end{gathered}
$$

which in particular admits the following "mass-Casimir" invariant

$$
\mathcal{C}=\Pi_{0}^{2}-\Pi_{1}^{2}+2 H N \Pi_{1}
$$

The Poisson brackets between conserved translation charges and coordinates define a symplectic structure

$$
\begin{array}{ll}
\left\{\Pi_{0}, x^{0}\right\}=1, & \left\{\Pi_{0}, x^{1}\right\}=-H x^{1}, \\
\left\{\Pi_{1}, x^{0}\right\}=0, & \left\{\Pi_{1}, x^{1}\right\}=1,
\end{array}
$$

which we anticipated in (3).

The Casimir relation (34) for the conserved charges leads to the dS-spacetime mass-shell condition by substituting the expression, (29) and (30), of the conserved charges $N$, $\Pi_{\alpha}$ in terms of the physical momenta $p_{\alpha}$ and coordinates $x^{\beta}$ :

$$
\left(p_{0}\right)^{2}-\left(p_{1}\right)^{2} e^{-2 H x^{0}}=m^{2} .
$$

In particular one has $p_{0}=\left|p_{1}\right| e^{-H x^{0}}$ for massless particles.

Note that we restrict our focus on the case of negative $p_{1}$ (so that $\dot{x}^{1} / \dot{x}^{0}$ is positive) and therefore take $p_{0}=$ $-p_{1} e^{-H x^{0}}$ as we already showed in 5 .

The evolution of coordinates $x^{\mu}$ along a particle worldline with parameter $\tau$ can be obtained in manifestly covariant form by using a standard Hamiltonian setup with (37) playing the role of Hamiltonian:

$$
\begin{aligned}
& \dot{x}^{1}=\left\{\left(p_{0}\right)^{2}-e^{-2 H x^{0}}\left(p_{1}\right)^{2}, x^{1}\right\}=-2 e^{-2 H x^{0}} p_{1} \\
& \dot{x}^{0}=\left\{\left(p_{0}\right)^{2}-e^{-2 H x^{0}}\left(p_{1}\right)^{2}, x^{0}\right\}=2 p_{0}=2\left|p_{1}\right| e^{-H x^{0}} .
\end{aligned}
$$

Then the worldline of a massless particle with initial conditions $x^{1}(\tau=0)=\bar{x}^{1}$ and $x^{0}(\tau=0)=\bar{x}^{0}$ reads:

$$
x^{1}\left(x^{0}\right)-\bar{x}^{1} \equiv \int_{\bar{x}^{0}}^{x^{0}} \frac{\dot{x}^{1}}{\dot{x}^{0}} d x^{0}=\left(\frac{e^{-H \bar{x}^{0}}-e^{-H x^{0}}}{H}\right) \text {. }
$$

In particular for $\bar{x}^{1}=\bar{x}^{0}=0$ this gives a wordline of the form already anticipated in Eq. (4) of Subsection II.A.

A convenient way to expose the effects of redshift can be based on the comparison of measurements of the same particle made by two different observers. For this we need to explicitate the form of finite translation transformations.

The action of the symmetries generators on the phase space functions is represented through the ordinary left action of Lie groups

$$
G_{\mathbf{v}} \triangleright A(\xi)=\sum_{n=0}^{\infty} \frac{1}{n !} \underbrace{\{\mathbf{v} \cdot \mathfrak{g}, \ldots\{\mathbf{v} \cdot \mathfrak{g}}_{n \text { times }}, A(\xi)\} \ldots\} .
$$

being $G_{\mathbf{v}}$ an element of the group identified by the vector parameter $\mathbf{v}$, and $\mathfrak{g}$ a set of elements of the algebra (the generators of the symmetry transformation associated to the group element $G_{v}$ ). For example a generic translation $\mathcal{T}_{a}$, connected to the time and space translation generators $\overrightarrow{\mathfrak{t}}=\left(\mathfrak{t}_{0}, \mathfrak{t}_{1}\right)$ by the translation parameters $\vec{a}=\left(a^{0}, a^{1}\right)$, acts on a generic phase space function $F(\xi)$ as

$$
\mathcal{T}_{a} \triangleright F(\xi)=\sum_{n=0}^{\infty} \frac{1}{n !} \underbrace{\{\vec{a} \cdot \overrightarrow{\mathfrak{t}}, \ldots\{\vec{a} \cdot \overrightarrow{\mathfrak{t}}}_{n \text { times }}, F(\xi)\} \ldots\} .
$$

Then, translation transformations $\mathcal{T}_{a}$ in deSitter spacetime act in the following way on spacetime coordinates and physical momenta:

$$
\begin{aligned}
& p_{0}^{B}=\mathcal{T}_{a} \triangleright p_{0}^{A}=p_{0}^{A} \\
& p_{1}^{B}=\mathcal{T}_{a} \triangleright p_{1}^{A}=p_{1}^{A} e^{-a^{0} H} \\
& x_{B}^{0}=\mathcal{T}_{a} \triangleright x_{A}^{0}=x_{A}^{0}-a^{0} \\
& x_{B}^{1}=\mathcal{T}_{a} \triangleright x_{A}^{1}=e^{a^{0} H}\left(x_{A}^{1}-\frac{a^{1}}{a^{0}} \frac{1-e^{-a^{0} H}}{H}\right)
\end{aligned}
$$

where $a^{0}$ and $a^{1}$ are, respectively, time and space translation parameters connecting the two observers. Therefore, if an observer Alice observes the following worldline of a photon emitted at her origin $\left(\bar{x}_{A}^{0}=0, \bar{x}_{A}^{1}=0\right)$ :

$$
x_{A}^{1}\left(x^{0}\right)=\left(\frac{1-e^{-H x^{0}}}{H}\right),
$$


then a second observer Bob, connected to Alice by a translation transformation, will observe the worldline

$$
x_{B}^{1}\left(x^{0}\right)+\frac{a^{1}}{a^{0}} \frac{e^{a^{0} H}-1}{H}=\left(\frac{e^{H a^{0}}-e^{-H x_{B}^{0}}}{H}\right) .
$$

From this one sees that the family of observers reached in their spacetime origin by the signal emitted in Alice's spacetime origin $\left(x_{B}^{1}\left(x_{B}^{0}=0\right)=0\right)$ are the ones connected to Alice by translations whose translation parameters obey the following simple relation

$$
a^{1}=a^{0} .
$$

We are at this point equipped to also highlight the aspects of relative momentum-space locality, i.e. the misleading inferences that can arise when the dS-spacetime observers adopt the $\Pi_{0}, \Pi_{1}$ coordinatization of momentum space. A key aspect of this is due to the fact that an emitter/observer Alice who measures a certain value of $p_{0}$ (determines $p_{0}$ "at Alice") also determines a corresponding value of $\Pi_{0}^{A}$ with $\Pi_{0}^{A}=p_{0}$, and $\Pi_{0}$ is a conserved charge so the value Alice assigns to $\Pi_{0}^{A}$ at some distant detector Bob still is given by the value of $p_{0}$ "at Alice". But translations, while acting trivially on $p_{0}$ as we saw in Eqs. 222 and 42 (but $p_{0}$ is not conserved along the worldline), act non-trivially on $\Pi_{0}^{A}$, so Alice's inference for the value of $\Pi_{0}$ at Bob actually disagrees from what Bob determines for $\Pi_{0}$ :

$$
\begin{aligned}
& \Pi_{0}^{B}=\mathcal{T}_{a} \triangleright \Pi_{0}^{A}=\Pi_{0}^{A}+\frac{a^{1}}{a^{0}} \Pi_{1}^{A}\left(1-e^{-H a^{0}}\right) \\
& \Pi_{1}^{B}=\mathcal{T}_{a} \triangleright \Pi_{1}^{A}=e^{-H a^{0}} \Pi_{1}^{A},
\end{aligned}
$$

as we already anticipated in $(19)$ and $(20)$. From these equations it follows that

$$
\Pi_{0}^{B}=e^{-a^{0} H} \Pi_{0}^{A} .
$$

Notice that all this is fully consistent with the fact that for both observers the value of $\Pi_{0}$ (conserved on the worldline) coincides with the value of $p_{0}$ when the worldline crosses the observer's spacetime origin: $\Pi_{0}=$ $\left.p_{0}\right|_{x^{0}=0, x^{1}=0}$. Moreover of course both observers agree (though giving different values to these charges) with the relationship $\Pi_{0}=-\Pi_{1}$ we showed in (18) among charges on worldlines that cross their spacetime origin.

This reproduces the conceptual picture of dS spacetime given in the previous sections. In order to also confirm the quantification of effects given in the previous sections we can now consider the case in which two massless particles are emitted at Alice, one at time $\bar{x}_{A}^{0}=0$ and the other one at time $\tilde{x}_{A}^{0}=\Delta x_{A}^{0}$, both with energy $p_{0}^{A}$. As second observer, Bob, we take one such that the first particle worldline crosses the osberver's spacetime origin, so that this particle's worldline is described in Bob's coordinatization by

$$
x_{B}^{1}=\frac{1-e^{-H x_{B}^{0}}}{H},
$$

where we have used the relations 42 and 45 . The second particle crosses Bob's spatial origin at a time $\tilde{x}_{B}^{0} \neq 0$ and Bob describes its worldline as follows:

$$
\tilde{x}_{B}^{1}+\frac{a^{1}}{a^{0}} \frac{e^{a^{0} H}-1}{H}=\frac{e^{-H\left(\Delta x_{A}^{0}-a^{0}\right)}-e^{-H \tilde{x}_{B}^{0}}}{H} .
$$

Using again eq. 45 this can be written in the form:

$$
\tilde{x}_{B}^{1}=\frac{e^{-H \Delta x_{B}^{0}}-e^{-H \tilde{x}_{B}^{0}}}{H},
$$

with

$$
\Delta x_{B}^{0} \equiv-\frac{\ln \left(-1+e^{H a^{0}}+e^{H\left(a^{0}-\Delta x_{A}^{0}\right)}\right)}{H} .
$$

Especially for what concerns the values determined for energies it is useful to offer our analysis using a very explicit notation, capable of differentiating among different ways in which a certain observable could be determined: for quantities measured at Bob's spatial origin adopting Bob's coordinatization we use the subscripts $B @ B$; for quantities described within Alice's coordinatization but concerning inferences for values of observables at Bob's spatial origin we use the subscripts $A @ B$; for quantities measured at Alice's spatial origin adopting Alice's coordinatization we use the subscripts $A @ A$; for quantities described within Bob's coordinatization but concerning inferences for values of observables at Alice's spatial origin we use the subscripts $B @ A$.

Equipped with this notation we can quickly assess the situation. For the first particle, crossing Bob's spatial origin at time $x_{B @ B}^{0}=0$, we have the relation we explicitely showed in Fig. 1

$$
p_{0}^{B @ B}=p_{0}^{A @ B}=e^{-H x_{A @ B}^{0}} p_{0}^{A @ A},
$$

where the first equality holds because of the triviality of the action of translation transformations on $p_{0}$ and the second equality follows from (37). For the second particle, crossing Bob's spatial origin at time $\tilde{x}_{B @ B}^{0}=$ $\Delta x_{B}^{0}$, we have

$$
\tilde{p}_{0}^{B @ B}=\tilde{p}_{0}^{A @ B}=e^{-H \tilde{x}_{A @ B}^{0}} \tilde{p}_{0}^{A @ A}=e^{-H \tilde{x}_{A @ B}^{0}} p_{0}^{A @ A},
$$

where the last equality reflects our choice to focus on two particles with the same energy at Alice $\tilde{p}_{0}^{A @ A}=p_{0}^{A @ A}$.

Comparing the expression for $p_{0}^{B @ B}$ and $\tilde{p}_{0}^{B @ B}$, as anticipated in (6), we find:

$$
\tilde{p}_{0}^{B @ B}=p_{0}^{B @ B} e^{-H\left(\tilde{x}_{A @ B}^{0}-x_{A @ B}^{0}\right)}
$$

which evidently reproduces the last equation of Subsec. IIA since dS-spacetime translations are such that $p_{0}^{B @ B}=p_{0}^{A @ B}, \tilde{p}_{0}^{B @ B}=\tilde{p}_{0}^{A @ B}$ and $\tilde{x}_{A @ B}^{0}-x_{A @ B}^{0}=$
$\tilde{x}_{B @ B}^{0}-x_{B @ B}^{0}=\Delta x_{B}^{0}$. 


\section{DE SITTER MOMENTUM SPACE}

Our next task is to analyze the dual picture of dS momentum space. We start by describing the metric on $\mathrm{dS}$ momentum space, with the same structure of the metric on dS spacetime considered in the preceding section. So we have, as already noted in (7),

$$
d k^{2}=\left(d p_{0}\right)^{2}-e^{2 \ell p_{0}}\left(d p_{1}\right)^{2},
$$

and in matrix form

$$
\zeta^{\alpha \beta}=\left(\begin{array}{cc}
1 & 0 \\
0 & -e^{2 \ell p_{0}}
\end{array}\right), \quad \zeta_{\alpha \beta}=\left(\begin{array}{cc}
1 & 0 \\
0 & -e^{-2 \ell p_{0}}
\end{array}\right) .
$$

For the coordinatization of spacetime in this case allowing for curvature of momentum space we find convenient to start with the possibility of spacetime coordinates $\chi^{\mu}$ which generate translations on $\mathrm{dS}$ momentum space (in analogy with the $\Pi_{\mu}$ coordinatization of momentum space adopted for parts of our analysis of properties of dS spacetime). For these we have that

$$
\left\{\chi^{0}, \chi^{1}\right\}=\ell \chi^{1}
$$

And we shall describe spacetime symmetries of this case with dS momentum space in terms of charges/generators of space translation, time translation and boost governed by the following Poisson bracket: 3 .

$$
\begin{aligned}
& \left\{p_{1}, p_{0}\right\}=0 \\
& \left\{\mathcal{N}, p_{0}\right\}=p_{1}, \quad\left\{\mathcal{N}, p_{1}\right\}=\frac{1-e^{-2 \ell p_{0}}}{2 \ell}-\frac{\ell}{2}\left(p_{1}\right)^{2}
\end{aligned}
$$

These phase-space Poisson brackets are compatible with the Jacobi identities upon assuming that the Poisson brackets involving $\chi^{\mu}$ and $p_{\mu}$ satisfy (also see $(9 p)$

$$
\begin{aligned}
& \left\{p_{1}, \chi^{1}\right\}=-1, \quad\left\{p_{1}, \chi^{0}\right\}=\ell p_{1}, \\
& \left\{p_{0}, \chi^{1}\right\}=0, \quad\left\{p_{0}, \chi^{0}\right\}=-1 .
\end{aligned}
$$

Just like in the dS-spacetime case the possibilities $\Pi_{\mu}$ and $p_{\mu}$ for coordintizing momentum space are comparably (though complementarily) convenient, for the $\mathrm{dS}$ momentum-space case which we are now considering one can conveniently coordinatize spacetime either with the coordinates $\chi^{\mu}$ or with the following coordinates $x^{\mu}$ :

$$
\begin{aligned}
& x^{1} \equiv \chi^{1}, \quad x^{0} \equiv \chi^{0}+\ell \chi^{1} p_{1}, \\
& \left\{x^{\mu}, x^{\nu}\right\}=0 \\
& \left\{p_{\mu}, x^{\nu}\right\}=-\delta_{\mu}^{\nu}
\end{aligned}
$$

\footnotetext{
${ }^{3}$ Note that rules of action of boosts on momenta of the type here given in Eq. 59] have been independently of interest in the literature on the $\kappa$-poincaré Hopf algebra 28, 29, which indeed in one of the formalisms forwhich a connection with the possibility of dS momentum space had been made 30, 31.
}

The convenience of these spacetime coordinates resides mainly in the fact that translation transformations act trivially on them, as shown in 63.

Note that all the phase-space relations (57), (58), (60) and (63) here given for the case of $\mathrm{dS}$ momentum space are dual to the ones, shown in the previous section, that hold in the dS-spacetime case for conserved charges $\Pi_{\alpha}$ and spacetime coordinates $x^{\alpha}$ : they are obtained one from the other through the substitutions $H \leftrightarrow \ell, x^{\mu} \leftrightarrow$ $p_{\mu}$ and $\Pi_{\mu} \leftrightarrow \chi^{\mu}$.

In comparing results obtained with the two coordinatizations of spacetime suitable for theories with dS momentum space, $\chi^{\mu}$ and $x^{\mu}$, it can be useful to also take notice of the following two possible representations of our boost generator:

$$
\begin{aligned}
& \mathcal{N}=p_{1} \chi^{0}+\left(\frac{1-e^{-2 \ell p_{0}}}{2 \ell}+\frac{\ell}{2} p_{1}^{2}\right) \chi^{1} \\
& \mathcal{N}=p_{1} x^{0}+\left(\frac{1-e^{-2 \ell p_{0}}}{2 \ell}-\frac{\ell}{2} p_{1}^{2}\right) x^{1} .
\end{aligned}
$$

Once again the duality of these formulae with the representations (30) and (31) of the boosts on dS spacetime is easily seen through the exchange $\Pi_{\alpha} \leftrightarrow \chi^{\alpha}, p_{\beta} \leftrightarrow x^{\beta}$ and $H \leftrightarrow \ell$.

The mass-Casimir invariant of the algebra 59 is

$$
\mathcal{C}_{\ell}=\left(\frac{2}{\ell} \sinh \left(\frac{\ell p_{0}}{2}\right)\right)^{2}-e^{\ell p_{0}} p_{1}^{2}
$$

Therefore for a massless particle on the dS momentum space one has the on-shell $\left(\mathcal{C}_{\ell}=0\right)$ condition of the form 10:

$$
p_{1}\left(p_{0}\right)=\frac{1-e^{-\ell p_{0}}}{\ell}
$$

There have been several studies (see, e.g., Refs. 16, 20, 23]) of the implications of momentum-space curvature for the properties of spacetime worldlines and travel times. While these previous studies focused on results applicable at leading order in $\ell$, drawing from the strength of the duality here exposed we are now in position to do an analogous study of the implications of dS momentum space to all orders in $\ell$ (exact).

The dependence of a coordinates $\chi^{\alpha}$ on the worldline parameter $\tau$ can be again found using the condition of on-shellness 66 as Hamiltonian: $\frac{d \chi^{\mu}}{d \tau} \equiv \dot{\chi}^{\mu}=\left\{\mathcal{C}_{\ell}, \chi^{\mu}\right\}$. This leads to

$$
\begin{aligned}
& \dot{\chi}^{0}=\frac{1}{\ell}\left(e^{\ell p_{0}}-e^{-\ell p_{0}}\right)+\ell p_{1}^{2} e^{\ell p_{0}} \\
& \dot{\chi}^{1}=2 p_{1} e^{\ell p 0}
\end{aligned}
$$

which evidently implies

$$
\begin{aligned}
& \chi^{0}(\tau)=\bar{\chi}^{0}+\left(\frac{1}{\ell}\left(e^{\ell p_{0}}-e^{-\ell p_{0}}\right)+\ell p_{1}^{2} e^{\ell p_{0}}\right) \tau, \\
& \chi^{1}(\tau)=\bar{\chi}^{1}+\left(2 p_{1} e^{\ell p_{0}}\right) \tau,
\end{aligned}
$$


where $\bar{\chi}^{\mu}$ are the initial conditions.

Specializing to the case of massless particles and eliminating the parameter $\tau$ we find a wordline of the form (15):

$$
\chi^{1}=\bar{\chi}^{1}-\left(\chi^{0}-\bar{\chi}^{0}\right)
$$

which is independent on the particle's energy and momentum. Notice however that translations on coordinates act non trivially on both the coordinates $\chi^{\mu}$ and the momenta, as shown already in 16 :

$$
\begin{aligned}
& p_{0}^{B}=\mathcal{T}_{a} \triangleright p_{0}^{A}=p_{0}^{A} \\
& p_{1}^{B}=\mathcal{T}_{a} \triangleright p_{1}^{A}=p_{1}^{A} \\
& \chi_{B}^{0}=\mathcal{T}_{a} \triangleright \chi_{A}^{0}=\chi_{A}^{0}-a^{0}+a^{1} \ell p_{1} \\
& \chi_{B}^{1}=\mathcal{T}_{a} \triangleright \chi_{A}^{1}=\chi_{A}^{1}-a^{1}
\end{aligned}
$$

where again we denote by $a^{0}$ and $a^{1}$ the translation parameters connecting two different observers. From this we see that if Alice observes worldlines of the form 68

$$
\chi_{A}^{1}-\bar{\chi}_{A}^{1}=-\left(\chi_{A}^{0}-\bar{\chi}_{A}^{0}\right) .
$$

for a particle emitted at a point $\left(\bar{\chi}_{A}^{0}=\bar{\chi}^{0}, \bar{\chi}_{A}^{1}=\bar{\chi}^{1}\right)$, then a distant observer Bob will observe:

$$
\chi_{B}^{1}\left(p, \chi^{0}\right)-\bar{\chi}_{B}^{1}=-\left(\chi_{B}^{0}-\bar{\chi}_{B}^{0}\right),
$$

with $\bar{\chi}_{B}^{1}=\bar{\chi}^{1}-a^{1}$ and $\bar{\chi}_{B}^{0}=\bar{\chi}^{0}-a^{0}+a^{1} \ell p_{1}$.

So when using $\chi^{\mu}$ coordinates one has that the form of the worldline is energy-independent but the translation transformation is momentum dependent.

It is interesting to compare these findings to the ones using the coordinatization $x^{\mu}$. The description of the worldlines in terms of the $x^{\mu}$ is found by observing that

$$
\begin{aligned}
& \dot{x}^{0}=\left\{\mathcal{C}_{\ell}, x^{0}\right\}=\frac{1}{\ell}\left(e^{\ell p_{0}}-e^{-\ell p_{0}}\right)-\ell p_{1}^{2} e^{\ell p_{0}} \\
& \dot{x}^{1}=\left\{\mathcal{C}_{\ell}, x^{1}\right\}=2 p_{1} e^{\ell p 0} .
\end{aligned}
$$

From this, by integrating $\dot{x}^{1}$ on the worldline parameter $\tau$, we find

$$
\int_{\tau_{0}}^{\tau} \dot{x}^{1} d \tau=\int_{\bar{x}^{0}}^{x^{0}} \frac{\dot{x}^{1}}{\dot{x}^{0}} d x^{0}=-e^{\ell p 0}\left(x^{0}-\bar{x}^{0}\right)
$$

from which is follows that

$$
x^{1}-\bar{x}^{1}=-e^{\ell p 0}\left(x^{0}-\bar{x}^{0}\right),
$$

This shows that with the $x^{\mu}$ coordinates the form of the worldline of a massless particle is momentum dependent, and confirms Eq. 13), which in the case of a particle emitted in the observer's origin, $\bar{x}^{0}=\bar{x}^{1}=0$, reduces to Eq. (11). As we already noted in Eq. (14), in the $x^{\mu}$ coordinates translations act trivially:

$$
\begin{aligned}
& p_{0}^{B}=\mathcal{T}_{a} \triangleright p_{0}^{A}=p_{0}^{A} \\
& p_{1}^{B}=\mathcal{T}_{a} \triangleright p_{1}^{A}=p_{1}^{A} \\
& x_{B}^{0}=\mathcal{T}_{a} \triangleright x_{A}^{0}=x_{A}^{1}-a^{1} \\
& x_{B}^{1}=\mathcal{T}_{a} \triangleright x_{A}^{1}=x_{A}^{0}-a^{0}
\end{aligned}
$$

Then is easy to obtain that if Alice observes a worldline of the form

$$
x_{A}^{1}-\bar{x}_{A}^{1}=-e^{\ell p_{0}^{A}}\left(x_{A}^{0}-\bar{x}_{A}^{0}\right),
$$

the translated observer Bob will agree about the wordline expression in his coordinates,

$$
x_{B}^{1}-\bar{x}_{B}^{1}=-e^{\ell p_{0}^{B}}\left(x_{B}^{0}-\bar{x}_{B}^{0}\right) .
$$

with $\bar{x}_{B}^{1}=\bar{x}_{A}^{1}-a^{1}$ and $\bar{x}_{B}^{0}=\bar{x}_{A}^{0}-a^{0}$.

Summarizing the issue of the choice of spacetime coordinates we have that the form of the worldline of a massless particle is momentum independent when using the $\chi^{\mu}$ coordinates whereas it is momentum dependent when using the $x^{\mu}$ coordinates. But this difference is balanced by the other difference: translations transformations are momentum dependent when using the $\chi^{\mu}$ coordinates whereas they are momentum independent when using the $x^{\mu}$ coordinates.

Following again the logical line of the previous section, we also observe that the observers whose origin is crossed by a given massless particle's worldline must be connected (if in relative rest) by a translation with parameters $a^{1}, a^{0}$ linked by

$$
a^{1}=-e^{\ell p_{0}} a^{0}
$$

As in the previous section for dS spacetime, also in this dS-momentum-space case we are interested in comparing observations made by two observers connected by a translation transformations. While in the dS-spacetime case it proved useful to consider two particles emitted at different times with same energy, we find useful for the dS-momentum-space case to consider two particles emitted simultaneously with different energies.

So let us consider two massless particles emitted with different energies $p_{0}$ and $\tilde{p}_{0}$, in the origin of the observer Alice:

$$
\begin{aligned}
& x_{A}^{1}=-e^{\ell p_{0}} x_{A}^{0} \\
& x_{A}^{1}=-e^{\ell \tilde{p}_{0}} x_{A}^{0}
\end{aligned}
$$

Note that in the dS-momentum-space case the momenta are conserved along the motion and under invariant under translations, so we omit observer's indices for them in this section.

For a translated observer Bob such that the worldline energy $p_{0}$ intercepts his origin one has a description of the worldlines in terms of the following equations:

$$
x_{B}^{1}=-e^{\ell p_{0}} x_{B}^{0}
$$

and

$$
\tilde{x}_{B}^{1}=-e^{\ell \tilde{p}_{0}} \tilde{x}_{B}^{0}+e^{\ell p_{0}} a^{0}\left(1-e^{\ell\left(\tilde{p}_{0}-p_{0}\right)}\right)
$$

where we made use of the relation $(83)$. So the particle with energy $\tilde{p}_{0}$ arrives at Bob's spatial origin at time:

$$
\tilde{x}_{B}^{0}\left(\tilde{x}_{B}^{1}=0\right)=-a^{0}\left(1-e^{-\ell\left(\tilde{p}_{0}-p_{0}\right)}\right)
$$


As done at the end of the previous section, let us use again notation specifying the value of observables either at Alice or at Bob, and according to Alice's coordinatization of Bob's coordinatization. Accoding to Alice's coordinatization the particle of energy $p_{0}$ arrives in Bob's origin at time

$$
x_{A @ B}^{0}=a^{0}
$$

whereas the other particle is at Bob at time

$$
\tilde{x}_{A B B}^{0}=a^{0} e^{-\ell\left(\tilde{p}_{0}-p_{0}\right)} .
$$

In the particularly interesting case in which the energy $p_{0}$ is small enough that (within a given experimental sensitivity) the term $e^{\ell p_{0}}$ in 90 can be neglected (so the behaviour of that massless particle is as if momentum space was flat and ordinary special relativity was applicable), then one finds that (90) takes the form used for Fig. 2,

$$
\tilde{x}_{@ B}^{0}=a^{0} e^{-\ell \tilde{p}_{0}^{@ B}},
$$

For completeness let us also observe that from 89 and (90) it follows that in Alice's coordinatization the relation between the two arrival times at Bob's spatial origin is

$$
\tilde{x}_{A @ B}^{0}=x_{A @ B}^{0} e^{-\ell\left(\tilde{p}_{0}-p_{0}\right)},
$$

which is the equation (12) reported at the end of section IIB. And we also stress again that this is completely analogous to Eq. (53) for the dS-spacetime case, describing the relation between the energies inferred by Alice as values of energies at Bob for the case of two massless particles emitted with same energy but at different times.

\section{ASIDE ON THE NEWTON-WIGNER OBSERVABLE}

While, as shown above, it is not necessary for exposing the duality which was here of interest, there are interesting implicit roles in our analysis for the Newton-Wigner observable $\mathcal{A}$, defined by

$$
\mathcal{A}=\int d x^{1}-\dot{x}^{1} d \tau=\int d x^{1}-\frac{\dot{x}^{1}}{\dot{x}^{0}} d x^{0} .
$$

Let us start noticing that in the dS-spacetime case for massless particles the Hamiltonian constraint can be written as

$$
\mathcal{H}_{H}=p_{1}+p_{0} e^{H x^{0}},
$$

which in turn allows one to write the Newton-Wigner observable straightforwardly using $(38)$ and $(5)$ :

$$
\mathcal{A}_{H}=\int_{0}^{x^{0}} d x^{1}-e^{-H x^{0}} d x^{0}=x^{1}-\left(\frac{1-e^{-H x^{0}}}{H}\right) \text {. }
$$

Of course the Newton-Wigner observable commutes with the Hamiltonian constraint: $\left\{\mathcal{H}_{H}, \mathcal{A}_{H}\right\}=0$.

In the dS-momentum-space case we have that the Hamiltonian constraint takes the form 66 ,

$$
\mathcal{H}_{\ell}=p_{1}-\frac{1-e^{-\ell p_{0}}}{\ell},
$$

from which it follows that the Newton-Wigner observable can be written as

$$
\mathcal{A}_{\ell}=\int_{0}^{x^{0}} d x^{1}-e^{\ell p^{0}} d x^{0}=x^{1}+e^{\ell p^{0}} x^{0} .
$$

(And again on easily verifies that the NewtonWigner observable commutes with Hamiltonian constraint: $\left\{\mathcal{H}_{\ell}, \mathcal{A}_{\ell}\right\}=0$.)

A very efficient way to summarize the duality we here exposed between the dS-spacetime case and the dSmomentum-space case is contained in Eqs. (96), (97), (94) and (95), which can be nicely organized in the following table:

$$
\begin{array}{lc}
d S \text { momentum - space } & d S \text { spacetime } \\
\mathcal{H}_{\ell}=p_{1}-\frac{1-e^{-\ell p_{0}}}{\ell} & \mathcal{H}_{H}=p_{1}+p_{0} e^{H x^{0}} \\
\mathcal{A}_{\ell}=x^{1}+x^{0} e^{\ell p_{0}} & \mathcal{A}_{H}=x^{1}-\frac{1-e^{-H x^{0}}}{H},
\end{array}
$$

\section{CONCLUSIONS}

We feel we here provided a satisfactory understanding of the travel-time features and of some aspects of relative spacetime locality which had been encountered in previous studies of theories with curved momentum space, but for which a clear conceptual picture was still missing.

We expect it should be possible to use the results here reported as starting point for a similar understanding of other features and other manifestations of relative spacetime locality encountered in studies of theories with curved momentum spaces. Of particular interest from this perspective could be the results reported in Refs. [16, 24] on dual-gravity lensing and transverse relative locality. These arise from momentum spaces which do not have de Sitter geometry and produce novel effects and novel implications for spacetime locality along directions orthogonal to the one connecting the emitter and the detector (they do not affect the travel times but rather the directional information codified in the description of the relevant processes).

Concerning relative spacetime locality and its connection with manifestations of ordinary curved-spacetimeinduced as relativity of momentum-space locality our analysis here was facilitated by the fact that we 
have dealt exclusively with the idealized case of noninteracting particles. One can look back at our findings and abstract the observation that relative locality is a feature arising whenever the chosen coordinatization does not have simple properties under the relativisticsymmetry transformation of interest. In particular, for the translation transformations here considered relative locality arises when the coordinates have non-canonical Poisson brackets with the generators of translations. It is noteworthy that in the case of non-interacting particles one could always choose coordinates which are free from the relative-locality effects. Such coordinates are not always the most convenient (depending on what are the objectives on one's study) but they are always available in theories with only non-interacting particles. It appears that this aspect of simplicity should be lost for interacting particles in presence of curvature, or at least this is what is suggested by studies [16, 27] of the recently proposed "relative-locality framework" 7]. That framework allows to describe interactions compatibly with the presence of curvature on momentum space, but then the translation generators acquire a novel form such that one cannot provide for each (interacting) particle in the system a coordinatization with canonical Poisson brackets with the generators of translation transformations. It would be interesting to find an analogue of this feature of interacting theories with a curved momentum space in some theories with a curved spacetime.

This research was supported in part by the John Templeton Foundation.
[1] S. Majid, arXiv:hep-th/0006166, Lect. Notes Phys. 541 (2000) 227.

[2] G. Amelino-Camelia, arXiv:gr-qc/0012051, Int. J. Mod. Phys. D11 (2002) 35; arXiv:hep-th/0012238, Phys. Lett. B510 (2001) 255.

[3] J. Kowalski-Glikman, arXiv:hep-th/0207279, Phys. Lett. B547 (2002) 291.

[4] F. Girelli and E.R. Livine, arXiv:gr-qc/0412079 Braz. J. Phys. 35 (2005) 432.

[5] D. Ratzel, S. Rivera and F.P. Schuller, Phys. Rev. D83 (2011) 044047.

[6] L.N. Chang, Z. Lewis, D. Minic and T. Takeuchi, arXiv:1106.0068, Adv. High Energy Phys. 2011 (2011) 493514.

[7] G. Amelino-Camelia, L. Freidel, J. Kowalski-Glikman, L. Smolin, arXiv:1101.0931, Phys. Rev. D84 (2011) 084010.

[8] G. Amelino-Camelia, L. Freidel, J. Kowalski-Glikman and L. Smolin, arXiv:1106.0313, General Relativity and Gravitation 43 (2011) 2547.

[9] M. Born, Proc. R. Soc. Lond. A165 (1938) 291.

[10] Yu.A. Gol'fand, Soviet Physics JETP 10 (1960) 356; Soviet Physics JETP 16 (1963) 184.

[11] G. Amelino-Camelia and S. Majid, arXiv:hepth/9907110 Int. J. Mod. Phys. A15 (2000) 4301.

[12] C. Rovelli, arXiv:gr-qc/9710008, Living Rev.Rel. 1 (1998) 1.

[13] L. Smolin, Lect. Notes Phys. 669 (2005) 363.

[14] G. Amelino-Camelia, J. Ellis, N.E. Mavromatos, D.V. Nanopoulos and S. Sarkar, arXiv:astro-ph/9712103 Nature 393 (1998) 763.

[15] J. Ellis, N.E. Mavromatos and D.V. Nanopoulos, arXiv:0901.4052, Phys. Lett. B674 (2009) 83.
[16] L. Freidel and L. Smolin, arXiv:1103.5626

[17] A. Marcianó, G. Amelino-Camelia, N. R. Bruno, G. Gubitosi, G. Mandanici, A. Melchiorri, arXiv:1004.1110, JCAP 1006 (2010) 030.

[18] G. Amelino-Camelia, A. Marcianó, M. Matassa, G. Rosati, arXiv:1206.5315 Phys. Rev. D86 (2012) 124035.

[19] J. Kowalski-Glikman, G.Rosati, Relative Locality in Curved Space-time, arXiv:1303.7216

[20] G. Amelino-Camelia, M. Matassa, F. Mercati and G. Rosati, arXiv:1006.2126, Phys. Rev. Lett. 106 (2011) 071301.

[21] L. Smolin, arXiv:1007.0718

[22] M. Arzano, J. Kowalski-Glikman, arXiv:1008.2962, Class. Quant. Grav. 28 (2011) 105009.

[23] G. Amelino-Camelia, N. Loret and G. Rosati, arXiv:1102.4637. Phys. Lett. B700 (2011) 150.

[24] G. Amelino-Camelia, L. Barcaroli and N. Loret, arXiv:1107.3334, Int. J. of Theoret. Phys. 51 (2012) 3359.

[25] S. Hossenfelder, arXiv:1004.0418, Phys. Rev. Lett. 104 (2010) 140402.

[26] X. Calmet, S. Hossenfelder and R. Percacci, arXiv:1008.3345, Phys. Rev. D82 (2010) 124024.

[27] G. Amelino-Camelia, M. Arzano, J. Kowalski-Glikman, G. Rosati and G. Trevisan, arXiv:1107.1724. Class. Quantum Grav. 29 (2012) 075007.

[28] S. Majid and H. Ruegg, arXiv:hep-th/9405107, Phys. Lett. B334 (1994) 348.

[29] J. Lukierski, H. Ruegg, W. J. Zakrzewski, arXiv:hepth/9312153, Annals Phys. 243 (1995) 90.

[30] J. Kowalski-Glikman, arXiv:hep-th/0207279, Phys. Lett. B547 (2002) 291.

[31] G. Amelino-Camelia, arXiv:1003.3942, Symmetry 2 (2010) 230. 